\title{
Strongly-interacting massive particle and dark photon in the era of the intensity frontier
}

\author{
Ayuki Kamada $\odot,{ }^{1}$ Masaki Yamada $\odot,{ }^{2,3,4}$ and Tsutomu T. Yanagida ${ }^{5,6}$ \\ ${ }^{1}$ Center for Theoretical Physics of the Universe, Institute for Basic Science (IBS), \\ 55 Expo-ro, Yuseong-gu, Daejeon 34126, Korea \\ ${ }^{2}$ Frontier Research Institute for Interdisciplinary Sciences, Tohoku University, \\ Sendai, Miyagi 980-8578, Japan \\ ${ }^{3}$ Department of Physics, Tohoku University, Sendai, Miyagi 980-8578, Japan \\ ${ }^{4}$ Center for Theoretical Physics, Laboratory for Nuclear Science and Department of Physics, \\ Massachusetts Institute of Technology, Cambridge, Massachusetts 02139, USA \\ ${ }^{5}$ T. D. Lee Institute and School of Physics and Astronomy, Shanghai Jiao Tong University, \\ 800 Dongchuan Rd, Shanghai 200240, China \\ ${ }^{6}$ Kavli IPMU (WPI), UTIAS, The University of Tokyo, \\ 5-1-5 Kashiwanoha, Kashiwa, Chiba 277-8583, Japan
}

(Received 13 May 2020; accepted 11 September 2020; published 5 October 2020)

\begin{abstract}
A strongly interacting massive particle (SIMP) is an interesting candidate for dark matter (DM) because its self-interaction cross section can be naturally strong enough to address the astrophysical problem of small-scale structure formation. A simple model was proposed by assuming a monopole condensation, where composite SIMP comes from a "strongly interacting" U(1) d gauge theory. In the original model, the DM relic abundance is determined by the $3 \rightarrow 2$ annihilation process via the WessZumino-Witten term. In this paper, we discuss that the DM relic abundance is naturally determined also by a semiannihilation process via a kinetic mixing between the hypercharge gauge boson and the dark $U(1)_{d}$ gauge boson (dark photon). The dark photon can be discovered by LDMX-style missing momentum experiments in the near future.
\end{abstract}

DOI: $10.1103 /$ PhysRevD.102.075001

\section{INTRODUCTION}

The intensity frontier is one of the broad approaches to new physics in collider experiments and recently became more important as the Large Hadron Collider has not yet found a clear signal for new physics. We should also note the null results in direct-detection experiments of dark matter (DM), which may indicate that the mass of DM is not of order the electroweak or $\mathrm{TeV}$ scale. We therefore focus on the case in which the DM mass is in a sub-GeV region, which can be tested via rare events rather than by a direct production from high-energy particles. Among proposed high-intensity accelerators, the Light Dark Matter eXperiment (LDMX) [1] is designed to measure missing momentum in high-rate electron fixed-target reactions and can be a powerful discovery tool for such a light DM particle.

Published by the American Physical Society under the terms of the Creative Commons Attribution 4.0 International license. Further distribution of this work must maintain attribution to the author(s) and the published article's title, journal citation, and DOI. Funded by SCOAP ${ }^{3}$.
From the perspective of cosmology, the stronglyinteracting massive particle (SIMP) proposed in Refs. [2,3] naturally fits sub-GeV DM. They pointed out that the relic abundance of sub-GeV DM is consistent with the observed value if the $3 \rightarrow 2$ annihilation process dominates at the time of the freeze-out of DM and its cross section is determined by the mass scale of DM with an $\mathcal{O}(1)$ coupling. SIMPs can be naturally realized by composite particles like Standard Model (SM) pions. The $3 \rightarrow 2$ annihilation process is actually realized by the WessZumino-Witten term in the low-energy dark sector. Interestingly, the model predicts a self-interaction cross section of DM, which is potentially favored by the observations of small-scale structure in cosmology [4-8] (see Ref. [9] for a review). This is dubbed as the SIMP miracle. However, there is a difficulty in maintaining thermal equilibrium between the dark and visible sectors during the freeze-out of the $3 \rightarrow 2$ annihilation process, which is required for the SIMP miracle to work. This can be realized in rather complicated models like the ones proposed in Refs. [10-12] (see Refs. [13-20] for recent works).

In Ref. [21], we have proposed a simple model of the SIMP, where the composite DM "pions" consist of darksector "electrons" and "positrons" connected by a U(1) 
gauge interaction rather than a strong non-Abelian gauge interaction. We introduce a fundamental "monopole" for $\mathrm{U}(1)_{d}$ at a high-energy scale and assume a "monopole" condensation at the sub-GeV scale. It is not known how to write down the Lagrangian of this kind of theory including both a monopole and an electron, since the gauge field strength does not satisfy the Bianchi identity, and thus, the usual Lagrangian with the gauge boson is not viable. However, this does not mean that the theory does not exist. ${ }^{1}$ In fact, theories with "monopoles" and "electrons" have been extensively studied in $\mathcal{N}=2[25-28]$ and $\mathcal{N}=1$ supersymmetry [29-32] without specifying the Lagrangian. In this paper, we revisit our SIMP model and propose a scenario in which the DM relic abundance is determined by a $2 \rightarrow 2$ semiannihilation process [33] via the kinetic mixing between the $\mathrm{U}(1)_{\mathrm{d}}$ gauge boson and $\mathrm{U}(1)_{Y}$ gauge boson rather than the $3 \rightarrow 2$ annihilation process. The model is quite economical [34]; we do not need to introduce any other particles but just introduce dark-sector "electrons", a "monopole", and the $\mathrm{U}(1)_{\mathrm{d}}$ gauge boson (dark photon), the latter of which plays the roles of confinement and mediator to the visible sector. Although the SIMP miracle does not work in this scenario, the model is simple, and all small dimensionless parameters are expected to be naturally small due to nontrivial anomalous dimensions.

The detectability and testability of our model is quite different from other DM models. Since there is no "pion""pion"-photon interaction and the semiannihilation process is $p$ wave suppressed, it is very difficult to directly or indirectly detect the DM "pions." However, the kinetic mixing allows us to discover the dark photon by LDMX-like experiments. Our model is unique in the sense that it can be tested mainly by experiments designed to measure missing momentum in high-rate electron fixed-target reactions.

The organization of this paper is as follows. In the next section, we specify particle contents of our model at highand low-energy scales. We assume that the $\mathrm{U}(1)_{\mathrm{d}}$ gauge symmetry is spontaneously broken by a "monopole" condensation at the energy scale of $0.1-1 \mathrm{GeV}$, below which there are "pions." We calculate its self-interaction cross section and show that it is within the value potentially favored by the observations of small-scale structure. In Sec. III, we explain how the "pion" relic abundance is determined by the freeze-out process, taking into account a kinetic mixing between $\mathrm{U}(1)_{\mathrm{d}}$ and $\mathrm{U}(1)_{Y}$ gauge bosons. The relevant process is a semiannihilation, which shows the freeze-out qualitatively different but is quantitatively similar to the standard freeze-out via annihilation. We take all $\mathcal{O}(1)$ parameters to be within $(0.1,1)$ for a conservative calculation and present a consistent parameter space for the

\footnotetext{
${ }^{1}$ Although the local Lagrangian for an electron and a monopole is constructed in Ref. [22], it is not manifestly Lorentz invariant because of an arbitrary constant vector (see also, e.g., Refs. [23,24])
}

kinetic mixing parameter and the mass of the $\mathrm{U}(1)_{\mathrm{d}}$ gauge boson. Then, we discuss the condition that the $3 \rightarrow 2$ annihilation process is negligible in our calculation. Finally, we comment on the mixing between the SM Higgs boson and the "monopole." Section IV is devoted to conclusions.

\section{HIDDEN "PIONS" FROM A "MONOPOLE" CONDENSATION}

We introduce a scalar "monopole" $\phi$ and $N_{F}$ pairs of darksector "electrons" $\psi_{i}$ and "positrons" $\bar{\psi}_{i}$ with $\mathrm{U}(1)_{\mathrm{d}}$ gauge field [21]. To ensure the stability of "pions" in the low-energy dark sector, we assume $\mathrm{SU}\left(N_{F}\right)$ flavor symmetry under which the "electrons" and "positrons" transform in the fundamental and antifundamental representations, respectively. Then the "pions" transform as an adjoint representation and are therefore stable. The charge assignment for $\psi_{i}$ and $\bar{\psi}_{i}$ is summarized in Table I. We call the $\mathrm{U}(1)_{\mathrm{d}}$ gauge boson as a dark photon.

We consider the case where the $\mathrm{U}(1)_{\mathrm{d}}$ gauge symmetry is spontaneously broken by the "monopole" condensation in the low-energy dark sector, just like the Higgs mechanism [35]. Each pair of "electrons" and "positrons" is then confined and connected by a string formed by the "monopole" condensation [35] and composes mesons while there is no baryon state in the low-energy dark sector [36]. The string tension is determined by the energy scale of the "monopole" condensation, $\Lambda$, and sets the dynamical scale of the system. We assume the condensation of "electrons" and "positrons" that dynamically breaks the chiral symmetry and the "pions" are the lightest composite states in the low-energy dark sector. We also assume that the chiral symmetry for the "electrons" and "positrons" is only an approximate symmetry so that the mass of the "pions" is as large as (but smaller than) the condensation scale $\Lambda[3,37]$.

After the "monopole" condensation, there are $N_{\pi}=$ $N_{F}^{2}-1$ "pions," the radial component of "monopole," and a massive $\mathrm{U}(1)_{\mathrm{d}}$ gauge boson in the effective field theory. The "monopole" and the gauge boson are assumed to be heavier than the "pions," which we identify as DM.

There is only one energy scale in the dark sector, $\Lambda$, which is of order the masses of the "pions," "monopole," and dark photon denoted by $m_{\pi}, m_{\phi}$, and $m_{V}$, respectively. We introduce $\mathcal{O}(1)$ constants $c_{i}$ that represents our ignorance of an $\mathcal{O}(1)$ uncertainty in the low-energy effective field theory [37]. For example, we define $m_{\pi}=c_{\Lambda} \Lambda=$ $c_{m_{\phi}} m_{\phi}=c_{m_{V}} m_{V}$. We also introduce other $\mathcal{O}(1)$ parameters

TABLE I. Charge assignment for matter fields in the dark sector.

\begin{tabular}{cccc}
\hline \hline & $\mathrm{SU}\left(N_{F}\right)$ & $\mathrm{U}(1)_{d}$ & $\mathrm{U}(1)_{y}$ \\
\hline$\psi_{i}$ & $\square$ & 1 & 0 \\
$\bar{\psi}_{i}$ & $\square$ & -1 & 0 \\
\hline \hline
\end{tabular}


associated with interactions in the dark sector specified below. To calculate the conservative bounds, we take $c_{i} \in$ $(0.1,1)$ throughout this paper. In Ref. [21], we assumed $c_{i}=1$ for simplicity. However, these uncertainties are important to discuss the detectability of our model in collider experiments, like LDMX.

\section{A. Self-interactions}

The "pions" have self-interactions whose cross sections are determined by the size of "pions," which is of order $\Lambda^{-1}$. Representing an $\mathcal{O}(1)$ factor by $c_{1}$, we write the cross section as

$$
\begin{aligned}
\frac{\sigma_{\text {ela }}}{m_{\pi}} & =\frac{(4 \pi)^{4} c_{1}^{2} m_{\pi}}{4 \pi \Lambda^{4}} \\
& \simeq 2.7 \mathrm{~cm}^{2} / \mathrm{g}\left(\frac{c_{1} c_{\Lambda}^{2}}{(4 \pi)^{-1}}\right)^{2}\left(\frac{m_{\pi}}{100 \mathrm{MeV}}\right)^{-3}
\end{aligned}
$$

from the dimensional analysis. ${ }^{2}$ This is of order the upper bound on the self-interaction cross section of DM from the observations of cluster collisions, including the bullet cluster, ellipticity on Milky way, and cluster scales [39-43]. These constraints and discussions have $\mathcal{O}(1)$ uncertainties due to, say, the difficulties of numerical simulations, and hence, we consider that they are marginally consistent with $\sigma_{\text {ela }} / m_{\pi}=0.1-1 \mathrm{~cm}^{2} / \mathrm{g}$. The recent observations of smallscale structure potentially favors the self-interacting DM with a cross section of the same order [4-8,44]. We note that $m_{\pi}$ can be as small as about $10 \mathrm{MeV}$ if $c_{\Lambda}=c_{1}=0.1$.

\section{RELIC ABUNDANCE OF "PIONS"}

\section{A. Kinetic mixings and $\mathbf{2} \rightarrow \mathbf{2}$ semiannihilation process}

There must be a nonzero kinetic mixing $\epsilon$ between the $\mathrm{U}(1)_{\mathrm{d}}$ gauge boson and the $\mathrm{U}(1)_{Y}$ gauge boson because it is allowed by any symmetry [36]. There are two types of kinetic mixing terms in theories consisting simultaneously of both a "monopole" and an "electron": $\epsilon^{\prime} B_{\mu \nu} F^{\mu \nu}$ and $\epsilon B_{\mu \nu} \tilde{F}^{\mu \nu}$, where $B_{\mu \nu}$ and $F_{\mu \nu}$ are the field strengths of $\mathrm{U}(1)_{Y}$ and $\mathrm{U}(1)_{\mathrm{d}}$ gauge bosons, respectively, and $\tilde{F}^{\mu \nu} \equiv$ $(1 / 2) \varepsilon^{\mu \nu \rho \sigma} F_{\rho \sigma}$. If the $C P$ symmetry is conserved, either of these mixing terms is allowed. ${ }^{3}$ However, one may

\footnotetext{
${ }^{2}$ We assume $c_{1} c_{\Lambda}^{2} \lesssim(4 \pi)^{-1}$ throughout this paper so that the scattering cross section is less than the geometrical cross section, $4 \pi / m_{\pi}^{2}$, that is below the unitarity bound for $v<c$ [38].

${ }^{3}$ One may think that $\epsilon B_{\mu \nu} \tilde{F}^{\mu \nu}$ itself violates the $C P$ symmetry. In general, either of $F_{\mu \nu}$ and $\tilde{F}_{\mu \nu}$ can be chosen to be a tensor, and the other one is a pseudotensor. If we choose the definition in which $B_{\mu \nu}$ and $\tilde{F}_{\mu \nu}$ are tensors and $\tilde{B}_{\mu \nu}$ and $F_{\mu \nu}$ are pseudotensors, the kinetic mixing term $\epsilon B_{\mu \nu} \tilde{F}^{\mu \nu}$ conserves the $C P$ symmetry. In this case, dark "pions" transform as $\pi \rightarrow-\pi$ (rather than $\left.\pi \rightarrow-\pi^{\mathrm{T}}\right)$ under the $C P$, so that $\left[\operatorname{Tr}\left[\pi \partial_{\mu} \pi \partial_{\nu} \pi\right]-(\mu \leftrightarrow \nu)\right]$ is also a tensor and can be mixed with $\tilde{F}_{\mu \nu}$.
}

expect that the $C P$ symmetry is violated in the dark sector and both mixing terms are present in general.

The $\mathrm{U}(1)_{\mathrm{d}}$ gauge theory may be conformal in the presence of "monopole" as well as "electrons" [27,28], which implies that the gauge field strength $F_{\mu \nu}$ has an scaling dimension larger than 2 as is guaranteed by the unitarity bound [45]. As a result, the kinetic mixing terms are irrelevant operators and are suppressed at low energy [21], if present. This naturally results in small $\epsilon^{\prime}$ and $\epsilon$ in our model. Hereafter, we represent $B_{\mu \nu}$ as the photon field strength and absorbs the Weinberg angle into $\epsilon^{\prime}$ and $\epsilon$ for notational simplicity.

In this paper, we mainly consider the case with $\epsilon B_{\mu \nu} \tilde{F}^{\mu \nu}$ and without $\epsilon^{\prime} B_{\mu \nu} F^{\mu \nu}$ for simplicity unless otherwise stated. In the dual basis, our model looks similar to the standard spontaneously broken $\mathrm{U}(1)_{\mathrm{d}}$ gauge theory, where the $\mathrm{U}(1)_{\mathrm{d}}$ symmetry is (spontaneously) broken by the condensation of the "Higgs" field (i.e., the scalar "monopole" in the original basis) and the kinetic mixing term looks the same as the usual one, $\epsilon B_{\mu \nu} F^{\mu \nu}$. Then we can quote constraints on the kinetic mixing parameter to compare our result with the present and future constraints. We will explain the case only with $\epsilon^{\prime}$, which leads to a similar result to the case only with $\epsilon$.

Here, we note that $\tilde{F}_{\mu \nu}$ does not satisfy the Bianchi identity, $\varepsilon^{\mu \nu \rho \sigma} \partial_{\nu} \tilde{F}_{\rho \sigma}=0$, in theories consisting simultaneously of both a "monopole" and an "electron" (see, e.g., Ref. [46]). Then an operator mixing between $\tilde{F}_{\mu \nu}$ and $\operatorname{Tr}\left[\pi \partial_{\mu} \pi \partial_{\nu} \pi\right]$ is allowed in those theories. Therefore, once we allow the nonzero kinetic mixing, $\epsilon B^{\mu \nu} \tilde{F}_{\mu \nu}$, we can have a term like

$$
\mathcal{L} \supset c_{\epsilon} \frac{(4 \pi)^{2}}{\Lambda^{3}} \epsilon B^{\mu \nu} \operatorname{Tr}\left[\pi \partial_{\mu} \pi \partial_{\nu} \pi\right]
$$

where $c_{\epsilon}$ is an $\mathcal{O}(1)$ constant. This operator leads to a semiannihilation process of $\pi \pi \rightarrow \pi \gamma$ only in the presence of a "monopole" and "electrons." If $\tilde{F}_{\mu \nu}$ satisfied the Bianchi identity, one could write $\tilde{F}_{\mu \nu}=\partial_{\mu} \tilde{V}_{\nu}-\partial_{\nu} \tilde{V}_{\mu}$ with $\tilde{V}_{\mu}$ being a (magnetic) gauge field of $\mathrm{U}(1)_{\mathrm{d}}$. Then the kinetic mixing operator $B^{\mu \nu} \tilde{F}_{\mu \nu}$ could be written as $-2 \partial_{\mu} B^{\mu \nu} \tilde{V}_{\nu}=0$ after the integration by parts for the on shell photon. However, $\tilde{F}_{\mu \nu}$ does not satisfy the Bianchi identity in the presence of a "monopole" as well as "electrons". There is no reason that we prohibit the operator of Eq. (2) and the on shell photon is produced by the annihilation process, $\pi \pi \rightarrow \pi \gamma$.

The operator of Eq. (2) vanishes for $N_{\pi}<3$, since it is antisymmetric in the flavor $\mathrm{SU}\left(N_{F}\right)$, so that we assume $N_{F} \geq 2$ in our model. We note that the "pions" transform as an adjoint representation of the flavor $\mathrm{SU}\left(N_{F}\right)$. The two "pions" in the initial state must be antisymmetric in the flavor $\mathrm{SU}\left(N_{F}\right)$ to contact with the one "pion" in the final state. On the other hand, the initial state of the 
semiannihilation process must be symmetric in terms of the "pion" exchange because "pions" are bosons. These observations imply that the initial angular momentum must be antisymmetric and the semiannihilation process is $p$ wave suppressed. We thus expect that its cross section can be estimated as

$$
\langle\sigma v\rangle_{\pi \pi \rightarrow \pi \gamma} \sim c_{\epsilon}^{2} \epsilon^{2} \frac{(4 \pi)^{4} m_{\pi}^{4}}{4 \pi \Lambda^{6}}\left(\frac{T}{m_{\pi}}\right)
$$

where a factor of $c_{\epsilon}^{2} \epsilon^{2}(4 \pi)^{4} / \Lambda^{6}$ comes from Eq. (2) and the power of $m_{\pi}$ is determined by the dimensional analysis. We absorb an $\mathcal{O}(1)$ uncertainty into $c_{\epsilon}$. This interaction is in thermal equilibrium at a temperature higher than $m_{\pi}$ for $c_{\epsilon} \epsilon \gtrsim 4 \times 10^{-12} c_{\Lambda}^{-3}\left(m_{\pi} / 100 \mathrm{MeV}\right)^{1 / 2}$. The temperature of the "pions" is the same as that of the SM sector until the semiannihilation process freezes out at $T / m_{\pi} \sim 1 / 20$.

\section{B. Relic abundance}

As the temperature becomes lower than the "pion" mass, the number density of "pions" is suppressed by the Boltzmann factor and eventually the $\pi \pi \rightarrow \pi \gamma$ semiannihilation process freezes out. We note that the $\pi \pi \rightarrow \pi \gamma$ semiannihilation process is similar to but is slightly different from the standard annihilation process in the weakly interacting massive particle (WIMP) scenario. The important difference is that the "pion" in the final state can be relativistic and may heat the dark sector $[14,47,48]$. From the Boltzmann equation of the "pions," the evolution equations of the yield $Y_{\pi}\left(\equiv n_{\pi} / s\right)$ and the inverse temperature $x_{\pi}\left(\equiv m_{\pi} / T_{\pi}\right)$ are approximated as

$$
\begin{gathered}
\frac{d}{d x} Y_{\pi} \approx-\frac{\lambda}{x^{2}} Y_{\pi}^{2}, \\
x \frac{d}{d x}\left(\frac{x_{\pi}}{x}\right) \approx \frac{x_{\pi}}{x}+\frac{2}{3} \bar{\lambda} Y_{\pi}\left(\frac{x_{\pi}}{x}\right)^{2},
\end{gathered}
$$

for $\quad x \quad\left(\equiv m_{\pi} / T\right)>x_{\mathrm{FO}} \quad\left(\equiv m_{\pi} / T_{\mathrm{FO}}\right), \quad$ where $s=$ $\left(2 \pi^{2} / 45\right) g_{*} T^{3}, T$ is the temperature of the SM particles, and $T_{\mathrm{FO}}$ is the freeze-out temperature (see Ref. [47] for the original equations without using approximations). The effective number of relativistic degrees of freedom, $g_{*}$, is taken to be about 10. The dimensionless reaction rates are given by

$$
\lambda=\frac{x s\left\langle\sigma v_{\text {rel }}\right\rangle}{2 H}, \quad \bar{\lambda} \approx-(\gamma-1) \lambda,
$$

where $\gamma(=5 / 4)$ is the Lorentz factor that DM achieves through semiannihilation.

Assuming $x_{\mathrm{FO}} \sim 20$, we numerically solve Eqs. (4) and (5). The time evolutions of the yield and the temperature of "pions" are shown as black curves in Fig. 1, where the yield is normalized by $Y_{\pi}^{\mathrm{FO}} \equiv 2 x_{\mathrm{FO}} / \lambda\left(x_{\mathrm{FO}}\right)$. The red curve in the
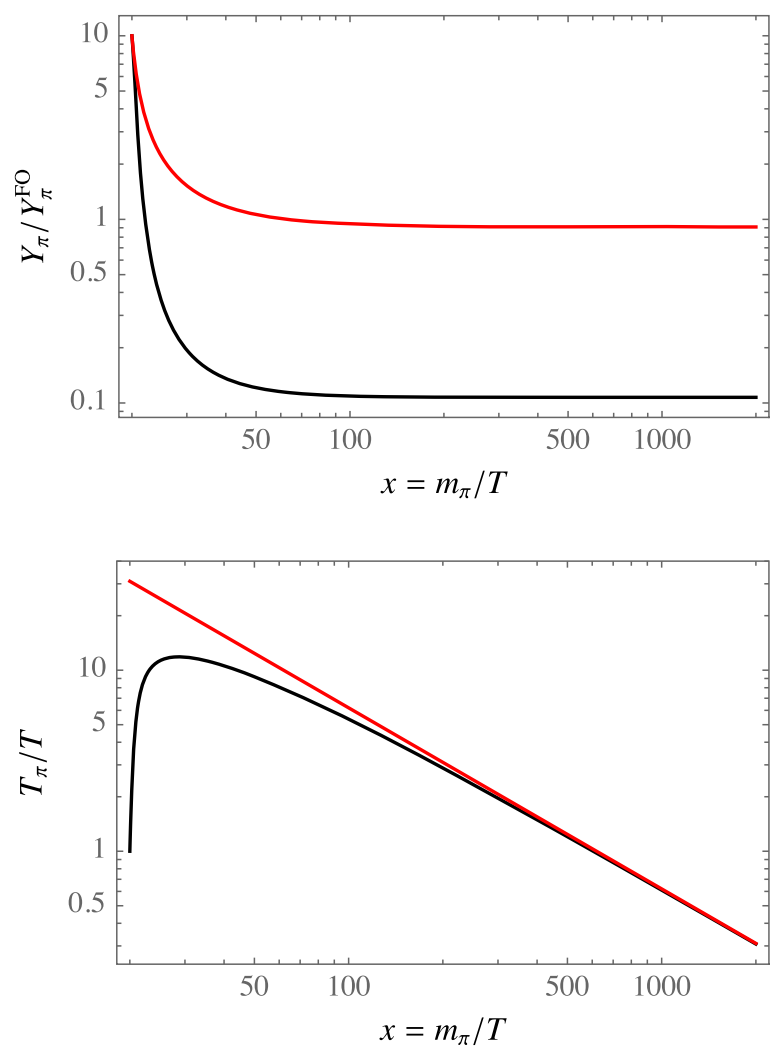

FIG. 1. Time evolutions of the yield $Y_{\pi}$ (black curve in the upper panel) and the temperature of "pions" $T_{\pi}$ (black curve in the lower panel) for the case of $x_{\mathrm{FO}}=20$. The red curve in the upper panel is the yield calculated in the case with $T_{\pi}=T$. The red curve in the lower panel is the asymptotic line of $30 T / T_{\mathrm{FO}}$.

upper panel is the one without the self-heating while that in the lower panel is $x_{\pi}=0.033 x^{2} / x_{\mathrm{FO}}$ to which the numerical result asymptotically approaches. Thus, we obtain the asymptotic value of the yield and the temperature of "pions" as

$$
Y_{\pi}^{\mathrm{FO}} \simeq c_{Y} \frac{2 x_{\mathrm{FO}}}{\lambda\left(x_{\mathrm{FO}}\right)}, \quad x_{\pi} \simeq c_{x} \frac{x^{2}}{x_{\mathrm{FO}}}
$$

for $x_{\pi} \gg x_{\mathrm{FO}}$, where $c_{Y}=\mathcal{O}(0.1)$ and $c_{x}=\mathcal{O}(0.1)$ are numerical constants. ${ }^{4}$ These results are different from the ones for the WIMP scenario by a factor of order 0.1. This is because the relativistic "pion" in the final state of the semiannihilation process heats the dark sector, which results in the relative increase for the $p$-wave semiannihilation rate. We do not determine the $\mathrm{O}(1)$ factors, since they are accurate enough for our purpose (we rely on the dimensional analysis). To determine them precisely, one

\footnotetext{
${ }^{4}$ The initial condition is taken to be $Y_{\pi}=c_{\mathrm{ini}} x_{\mathrm{FO}}^{2} / \lambda\left(x_{\mathrm{FO}}\right)$ and $x_{\pi}=x$ at $x=x_{\mathrm{FO}}$ with $c_{\text {ini }}$ being an $\mathcal{O}(1)$ constant. The numerical coefficients $c_{Y}$ and $c_{x}$ depend on $c_{\text {ini }}$ only logarithmically, while they linearly depend on $x_{\mathrm{FO}}^{-1}$.
} 


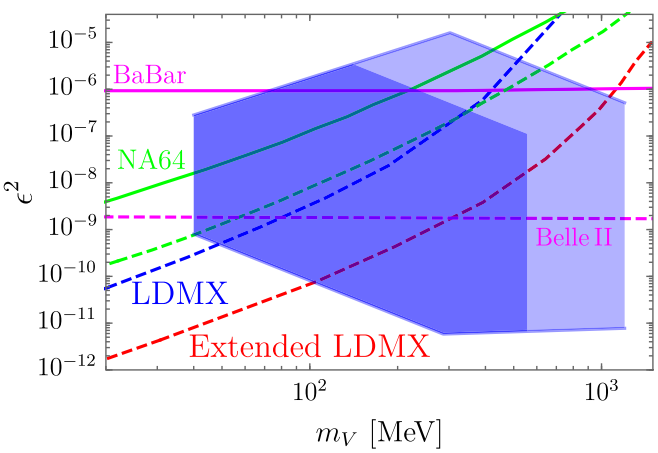

FIG. 2. Allowed region of the kinetic mixing parameter $\epsilon^{2}$. The shaded regions are parameters in which we obtain the correct DM relic abundance and $\sigma_{\text {ela }} / m_{\pi} \in(0.1,1) \mathrm{cm}^{2} / \mathrm{g}$. The magenta and green lines are the upper bound by the BABAR $[49,50]$ experiment and NA64 [51], respectively. The dashed lines are the expected sensitivities of Belle II (magenta) [52,53], NA64 (green) [54], LDMX (blue), and Extended LDMX (red) [1] experiments.

needs to incorporate an inverse semiannihilation process, which we neglect and will lead to a (not drastically) larger pion relic abundance. The energy density of the "pions" at present is consistent with the observed value of the DM relic density when

$$
\epsilon \sim 5 \times 10^{-7} c_{Y}^{1 / 2} c_{\epsilon}^{-1} c_{\Lambda}^{-3}\left(\frac{m_{\pi}}{100 \mathrm{MeV}}\right) .
$$

The kinetic mixing can be as large as, e.g., $\mathcal{O}\left(10^{-3}\right)$ for $m_{\pi}=100 \mathrm{MeV}$ if $c_{\Lambda}=c_{\epsilon}=0.1$.

The second term in the right-hand side of Eq. (5) becomes negligible after the freeze-out if the semiannihilation process is $p$ wave suppressed and $\bar{\lambda} \propto 1 / x_{\pi}$. Then, the temperature of the "pions" scales as $T_{\pi} \propto 1 / a^{2}$ just like the nonrelativistic matter and the DM "pions" are cold, where $a$ is the scale factor. This is in contrast to the case of a $s$-wave semiannihilation process discussed in Ref. [47], where it is found that $T_{\pi} \propto 1 / a$ because both the first and second terms in the right-hand side of Eq. (5) are relevant and are balanced until the self-interaction freezes out. In the latter case, the temperature of DM is not that small and DM is warm, which is tightly constrained by measurements of the Lyman- $\alpha$ forest [48]. On the other hand, the temperature of DM decreases faster, and DM is cold in our model.

We show the allowed region of the kinetic mixing parameter $\epsilon^{2}$ in Fig. 2. We assume that $c_{1}, c_{\Lambda}, c_{\epsilon} \in$ $(0.1,1)$ with a condition of $c_{1} c_{\Lambda}^{2}<(4 \pi)^{-1}$ (see footnote 3) for a conservative analysis, while we take $c_{Y}=0.1$ and $c_{m_{V}}=1 / 4$ for simplicity. The shaded regions are parameters in which the DM relic abundance can be consistent with the observed DM abundance and the self-interaction cross section can be $\sigma_{\text {ela }} / m_{\pi} \in(0.1,1) \mathrm{cm}^{2} / \mathrm{g}$. In the darkly shaded region, $\sigma_{\text {ela }} / m_{\pi}$ can be as large as $1 \mathrm{~cm}^{2} / \mathrm{g}$, while in the lightly shaded region it is smaller than $1 \mathrm{~cm}^{2} / \mathrm{g}$ but can be larger than $0.1 \mathrm{~cm}^{2} / \mathrm{g}$.
The upper-left corner of the shaded region is bounded by the condition that $c_{\Lambda}$ should not be smaller than about 0.1 in Eq. (8). In the upper-right (lower-left) corner of the figure, the self-interaction cross section of "pions" becomes too small (large) to be consistent with the observations of the small-scale structure. If $\epsilon^{2}$ is smaller than about $10^{-11}$ and $N_{\pi} \geq 5$, the $3 \rightarrow 2$ annihilation process becomes relevant during the freeze-out process as we will see shortly.

\section{Experimental constraints}

Since there is no $\pi-\pi-\gamma$ (or dark photon) interaction due to the flavor $\mathrm{SU}\left(N_{F}\right)$, the "pions" cannot be detected by the direct-detection experiments of DM. On the other hand, the dark photon can be produced via the kinetic mixing and can be discovered by some experiments employing missing momentum and/or energy techniques. In the figure, we plot the constraints on the kinetic mixing parameter by $B A B A R$ $[49,50,55]$ and NA64 $[51,56]$ in the magenta and green lines, respectively. We can see that most of the parameter space is consistent with the present upper bound. The expected sensitivities of future experiments are shown by the dashed lines for Belle II (magenta) [52,53], NA64 (green) [54], LDMX (blue), and Extended LDMX (red) [1] experiments. We find that the (Extended) LDMX experiment as well as Belle II experiment can cover a large parameter space.

Note that the dark photon cannot decay into two "pions" in our model. This implies that the dark photon cannot decay solely into the dark sector for the case of $m_{V}<3 m_{\pi}$. On the other hand, the dark photon dominantly decays into the dark "pions" for the case of $m_{V}>3 m_{\pi}$. The LDMX experiment is designed to measure missing momentum in this kind of process. As we hope to indirectly detect the DM particle by LDMX-like experiments, we assume $m_{V}=4 m_{\pi}$ $\left(>3 m_{\pi}\right)$, i.e., $c_{m_{V}}=1 / 4$, to plot the figure. We predict that $m_{V}$ is larger than about $30 \mathrm{MeV}$ because we require $m_{V}>3 m_{\pi}$ and $m_{\pi} \gtrsim 10 \mathrm{MeV}$.

Here, we comment on the case in which there is only the other kinetic mixing term $\epsilon^{\prime} B^{\mu \nu} F_{\mu \nu}$ rather than $\epsilon B^{\mu \nu} \tilde{F}_{\mu \nu}$. In this case, Eq. (2) should be replaced by a term like $c_{\epsilon^{\prime}}(4 \pi)^{2} / \Lambda^{3} \epsilon \tilde{B}^{\mu \nu} \operatorname{Tr}\left[\pi \partial_{\mu} \pi \partial_{\nu} \pi\right]$ though our analysis of the semiannihilation process does not change much. The SM charged particles cannot emit on shell dark photons while the dark-sector particles can be produced via the off shell (dark) photons via the kinetic mixing. We expect that the cross section of such a process with missing particles is then given by the replacements of $m_{V}$ by $\Lambda$ and $\epsilon^{2}$ by $\epsilon^{\prime 2}$ with an additional factor of $N_{F} \alpha_{D} /(2 \pi) \ln (E / \Lambda)(\sim \mathcal{O}(1))$ for $E \gtrsim \Lambda$, where $E(=\mathcal{O}(1) \mathrm{GeV})$ is the energy of the scattering process $[50]{ }^{5}$ We note that the additional factor is just an $\mathcal{O}(1)$ factor and the difference between $m_{V}$ and $\Lambda$ is

\footnotetext{
${ }^{5}$ One may think that the cross section is dominated by a lowenergy contribution near the threshold of $3 m_{\pi}$ [50]. In our case, however, it is negligible due to the $p$-wave suppression effect.
} 
also an $\mathcal{O}(1)$ factor. We may absorb these factors into $c_{\epsilon}$ and $c_{m_{V}}$, respectively. Then the result is similar to the one shown in Fig. 2 with $\epsilon^{2} \rightarrow \epsilon^{\prime 2}$. Even in the presence of both kinetic terms, the result does not change much because their effects are additive for the production process in the experimental setups as well as for the semiannihilation process.

We also comment on the region near the lower bound on the "pion" mass $(\sim 10 \mathrm{MeV})$. As the "pions" are nonrelativistic and are suppressed by the Boltzmann factor during the freeze-out process of neutrinos, the effect of "pion" decoupling is almost negligible for observables such as the effective number of neutrinos. However, it is argued that its effect can be detected in the near future by the Simons Observatory [57] and CMB-S4 [58,59] if the "pion" mass is as small as about 10-15 MeV [60].

Finally, we note that the constraint from the indirect detection experiments of DM is not relevant in our model because the semiannihilation process is $p$ wave suppressed and is not efficient in the galactic scale (see, e.g., Ref. [61]).

\section{D. $3 \rightarrow 2$ annihilation process}

The "pions" may experience a $3 \rightarrow 2$ annihilation process via the following operator:

$$
c_{\mathrm{WZW}} \frac{(4 \pi)^{3}}{N^{3 / 2} \Lambda^{5}} \varepsilon^{\mu \nu \rho \sigma} \operatorname{Tr}\left[\pi \partial_{\mu} \pi \partial_{\nu} \pi \partial_{\rho} \pi \partial_{\sigma} \pi\right] .
$$

This term is allowed by any symmetry and is an analogy to the Wess-Zumino-Witten term in strong $\mathrm{SU}(\mathrm{N})$ gauge theories. It trivially vanishes for $N_{\pi}<5$, namely $N_{F}<3$. The cross section for the $3 \rightarrow 2$ annihilation process is calculated as [3]

$$
\left\langle\sigma v^{2}\right\rangle_{3 \rightarrow 2}=\frac{(4 \pi)^{6} c_{\mathrm{WZW}}^{2} 375 \sqrt{5} m_{\pi}^{5}}{2 \pi N_{F} \Lambda^{10}} \frac{T^{2}}{m_{\pi}^{2}} .
$$

We should check that it is not efficient during the freeze-out of the $2 \rightarrow 2$ semiannihilation process induced by Eq. (2). The condition is written as

$$
\left\langle\sigma v^{2}\right\rangle_{3 \rightarrow 2}\left(n_{\pi}^{\mathrm{eq}}\left(T_{\mathrm{FO}}\right)\right)^{2} \lesssim\langle\sigma v\rangle_{\pi \pi \rightarrow \pi \gamma} n_{\pi}^{\mathrm{eq}}\left(T_{\mathrm{FO}}\right) \simeq H\left(T_{\mathrm{FO}}\right) .
$$

This condition is satisfied when

$$
\epsilon \gtrsim 2 \times 10^{-6} c_{\epsilon}^{-1}\left(\frac{c_{\mathrm{WZW}}}{0.1}\right)^{3 / 5}\left(\frac{m_{\pi}}{100 \mathrm{MeV}}\right)^{1 / 10},
$$

where we consider the case in which the relic abundance of "pions" is consistent with the observed DM abundance. In Fig. 2, the shaded region satisfies this condition with $c_{\mathrm{WZW}}=0.1$ and $c_{\epsilon}=1$. However, we note that Eq. (9) trivially vanishes and the constraint of Eq. (11) is not applied for the case of $N_{F}=2\left(N_{\pi}=3\right)$, which is the minimal case for semiannihilation to work in our model.
We also note that Eq. (2) leads to a $3 \rightarrow 2$ semiannihilation process [62] such as $\pi \pi e \rightarrow \pi e$, where $e$ generically represents the SM charged particles. The cross section of this process is roughly estimated as $\left(\sigma v^{2}\right)_{\pi \pi e \rightarrow \pi e} \sim(\sigma v)_{\pi \pi \rightarrow \pi \gamma}(4 \pi \alpha) / m_{\pi}^{3}$. Here, $\left\langle\sigma v^{2}\right\rangle_{\pi \pi e \rightarrow \pi e} n_{e}$ is suppressed by a factor of order $4 \pi \alpha n_{e} / m_{\pi}^{3}$ compared with $\langle\sigma v\rangle_{\pi \pi \rightarrow \pi \gamma}$. This is as small as $10^{-6}$ at the time of freeze-out $\left(n_{e} \sim T^{3}\right)$ and much smaller by many orders of magnitude at the present epoch, so that the process is not relevant for setting the relic abundance nor leading indirectdetection signals. Since $(\sigma v)_{\pi \pi \rightarrow \pi \gamma} \sim(v / c)^{2} 10^{-9} / \mathrm{GeV}^{2}$, $\left(\sigma v^{2}\right)_{\pi \pi e \rightarrow \pi e}$ is many orders of magnitude smaller than the one predicted in the model of Ref. [62]. Therefore, the process is not relevant for leading direct-detection signals.

\section{E. Mixing between the SM Higgs boson and the "monopole"}

There must be a nonzero mixing between the "monopole" $\phi$ and the SM Higgs field $H$ because the following interaction term is allowed by any symmetry:

$$
V_{\text {mix }}=\lambda|\phi|^{2}|H|^{2},
$$

where $\lambda$ is a constant. After the Higgs and "monopole" condensations, the mixing angle between the "monopole" and the SM Higgs field is given by

$$
\theta \simeq 0.023 \lambda c_{\operatorname{mix}}\left(\frac{m_{\pi}}{1 \mathrm{GeV}}\right)\left(\frac{m_{V}}{3 m_{\pi}}\right)
$$

where we assume that the "monopole"-condensation scale is related to $m_{\pi}$ by an $\mathcal{O}(1)$ factor $c_{\text {mix }}$.

There is a strong collider constraint on the mixing parameter from the Higgs-decay channel into two "monopoles" [63]. The "monopoles" can decay into muons after they are produced from the Higgs decay [64]. In this case, the branching ratio of the Higgs decay into the "monopoles" must be smaller than about $1 \%$ [65], which requires that the quartic coupling $\lambda$ must be smaller than of order $10^{-3}$. Such a small coupling may be naturally realized in our model because our model may be conformal above the "monopole" and "electron" mass scale, and the "monopole" has a relatively large anomalous dimension [27,28]. The search for the Higgs decay into muons may also be an interesting direction to test our model in the near future.

\section{CONCLUSIONS}

We revisited our SIMP model with dark-sector "electrons" and a "monopole" in U(1) gauge theory, motivated by the small-scale crisis in cosmology. We assumed a "monopole" condensation, which results in the formation of "pions" in the low-energy sector. The relic abundance of the "pions" is determined by the freeze-out process of semiannihilation, $\pi \pi \rightarrow \pi \gamma$, that is induced from a kinetic 
mixing between the $\mathrm{U}(1)_{\mathrm{d}}$ and $\mathrm{U}(1)_{Y}$ gauge bosons. We note that the on shell photon can couple to the dark sector through the mixing with the $\mathrm{U}(1)_{\mathrm{d}}$ gauge boson, since the $\mathrm{U}(1)_{\mathrm{d}}$ field strength does not satisfy the Bianchi identity. The very kinetic mixing allows us to discover the $\mathrm{U}(1)_{\mathrm{d}}$ gauge boson by LDMX-style missing momentum experiments in a large parameter space.

We note that the model is quite economical: the $\mathrm{U}(1)_{\mathrm{d}}$ gauge boson plays the roles of confinement and the mediator for the annihilation of "pions." The number of flavor $N_{F}$ can be as small as 2 to introduce an operator for the semiannihilation process. We assume $\mathrm{SU}\left(N_{F}\right)$ flavor symmetry to ensure the stability of "pions." One can promote this flavor symmetry to a gauge symmetry without changing our scenario qualitatively if the gauge coupling constant is small enough.

\section{ACKNOWLEDGMENTS}

T. T. Y. deeply thanks the experimental groups at TDLI for the discussions on the search for the dark photon. Without the discussion, we could not have reached the conclusion in this paper. We thank K. Yonekura for useful discussion. A. K. was supported by Institute for Basic Science under the Project Code No. IBS-R018-D1. M. Y. was supported by Leading Initiative for Excellent Young Researchers, MEXT, Japan. T. T. Y. was supported in part by the China Grant for Talent Scientific Start-Up Project and the JSPS Grant-in-Aid for Scientific Research Grants No. 16H02176, No. 17H02878, and No. 19H05810 and by the World Premier International Research Center Initiative (WPI Initiative), MEXT, Japan. M. Y. thanks the hospitality during his stay at DESY. T. T. Y. thanks Kavli IPMU for their hospitality recently.
[1] T. Åkesson et al. (LDMX Collaboration), arXiv:1808 .05219 .

[2] Y. Hochberg, E. Kuflik, T. Volansky, and J. G. Wacker, Phys. Rev. Lett. 113, 171301 (2014).

[3] Y. Hochberg, E. Kuflik, H. Murayama, T. Volansky, and J. G. Wacker, Phys. Rev. Lett. 115, 021301 (2015).

[4] D. N. Spergel and P. J. Steinhardt, Phys. Rev. Lett. 84, 3760 (2000).

[5] W. J. G. de Blok, Adv. Astron. 2010, 1 (2010).

[6] M. Boylan-Kolchin, J. S. Bullock, and M. Kaplinghat, Mon. Not. R. Astron. Soc. 415, L40 (2011).

[7] J. Zavala, M. Vogelsberger, and M. G. Walker, Mon. Not. R. Astron. Soc. 431, L20 (2013).

[8] A. Kamada, M. Kaplinghat, A. B. Pace, and H.-B. Yu, Phys. Rev. Lett. 119, 111102 (2017).

[9] S. Tulin and H.-B. Yu, Phys. Rep. 730, 1 (2018).

[10] H. M. Lee and M.-S. Seo, Phys. Lett. B 748, 316 (2015).

[11] N. Bernal, X. Chu, C. Garcia-Cely, T. Hambye, and B. Zaldivar, J. Cosmol. Astropart. Phys. 03 (2016) 018.

[12] Y. Hochberg, E. Kuflik, and H. Murayama, J. High Energy Phys. 05 (2016) 090.

[13] K. Tsumura, M. Yamada, and Y. Yamaguchi, J. Cosmol. Astropart. Phys. 07 (2017) 044.

[14] A. Kamada, H. Kim, and T. Sekiguchi, Phys. Rev. D 96, 016007 (2017).

[15] S.-Y. Ho, T. Toma, and K. Tsumura, J. High Energy Phys. 07 (2017) 101.

[16] S.-M. Choi, Y. Hochberg, E. Kuflik, H. M. Lee, Y. Mambrini, H. Murayama, and M. Pierre, J. High Energy Phys. 10 (2017) 162.

[17] A. Berlin, N. Blinov, S. Gori, P. Schuster, and N. Toro, Phys. Rev. D 97, 055033 (2018).

[18] S.-M. Choi, H. M. Lee, P. Ko, and A. Natale, Phys. Rev. D 98, 015034 (2018).

[19] M. Heikinheimo, K. Tuominen, and K. Langæble, Phys. Rev. D 97, 095040 (2018).
[20] Y. Hochberg, E. Kuflik, R. Mcgehee, H. Murayama, and K. Schutz, Phys. Rev. D 98, 115031 (2018).

[21] A. Kamada, M. Yamada, T. T. Yanagida, and K. Yonekura, Phys. Rev. D 94, 055035 (2016).

[22] D. Zwanziger, Phys. Rev. D 3, 880 (1971).

[23] K. A. Milton, Rep. Prog. Phys. 69, 1637 (2006).

[24] J. Terning and C. B. Verhaaren, J. High Energy Phys. 12 (2019) 152.

[25] N. Seiberg and E. Witten, Nucl. Phys. B426, 19 (1994); B430, 485(E) (1994).

[26] N. Seiberg and E. Witten, Nucl. Phys. B431, 484 (1994).

[27] P. C. Argyres and M. R. Douglas, Nucl. Phys. B448, 93 (1995).

[28] P. C. Argyres, M. R. Plesser, N. Seiberg, and E. Witten, Nucl. Phys. B461, 71 (1996).

[29] S. Giacomelli, J. High Energy Phys. 01 (2015) 044.

[30] S. Bolognesi, S. Giacomelli, and K. Konishi, J. High Energy Phys. 08 (2015) 131.

[31] D. Xie and K. Yonekura, Phys. Rev. Lett. 117, 011604 (2016).

[32] M. Buican and T. Nishinaka, Phys. Rev. D 94, 125002 (2016).

[33] F. D'Eramo and J. Thaler, J. High Energy Phys. 06 (2010) 109.

[34] M. Yamada, T. T. Yanagida, and K. Yonekura, Phys. Rev. Lett. 116, 051801 (2016).

[35] Y. Nambu, Phys. Rev. D 10, 4262 (1974).

[36] M. Yamada, T. T. Yanagida, and K. Yonekura, Phys. Lett. B 759, 459 (2016).

[37] M. Hansen, K. Langæble, and F. Sannino, Phys. Rev. D 92 , 075036 (2015).

[38] K. Griest and M. Kamionkowski, Phys. Rev. Lett. 64, 615 (1990).

[39] M. Markevitch, A. H. Gonzalez, D. Clowe, A. Vikhlinin, W. Forman, C. Jones, S. Murray, and W. Tucker, Astrophys. J. 606, 819 (2004). 
[40] D. Clowe, A. Gonzalez, and M. Markevitch, Astrophys. J. 604, 596 (2004).

[41] S. W. Randall, M. Markevitch, D. Clowe, A. H. Gonzalez, and M. Bradač, Astrophys. J. 679, 1173 (2008).

[42] M. Rocha, A. H. G. Peter, J. S. Bullock, M. Kaplinghat, S. Garrison-Kimmel, J. Oñorbe, and L. A. Moustakas, Mon. Not. R. Astron. Soc. 430, 81 (2013).

[43] A. H. G. Peter, M. Rocha, J. S. Bullock, and M. Kaplinghat, Mon. Not. R. Astron. Soc. 430, 105 (2013).

[44] F. Kahlhoefer, K. Schmidt-Hoberg, J. Kummer, and S. Sarkar, Mon. Not. R. Astron. Soc. 452, L54 (2015).

[45] G. Mack, Commun. Math. Phys. 55, 1 (1977).

[46] M. Blagojević and P. Senjanović, Phys. Rep. 157, 233 (1988).

[47] A. Kamada, H. J. Kim, H. Kim, and T. Sekiguchi, Phys. Rev. Lett. 120, 131802 (2018).

[48] A. Kamada, H. J. Kim, and H. Kim, Phys. Rev. D 98, 023509 (2018).

[49] B. Aubert et al. (BABAR Collaboration), in Proceedings, 34th International Conference on High Energy Physics (ICHEP 2008): Philadelphia, Pennsylvania (2008) [arXiv:0808.0017].

[50] E. Izaguirre, G. Krnjaic, P. Schuster, and N. Toro, Phys. Rev. D 91, 094026 (2015).

[51] D. Banerjee et al. (NA64 Collaboration), Phys. Rev. Lett. 123, 121801 (2019).
[52] W. Altmannshofer et al. (Belle-II Collaboration), Prog. Theor. Exp. Phys. 2019, 123C01 (2019); 2020, 029201 (E) (2020).

[53] G. Inguglia (Belle II Collaboration), Nuovo Cimento C 42 , 257 (2020).

[54] J. Beacham et al., J. Phys. G 47, 010501 (2020).

[55] J. Lees et al. (BABAR Collaboration), Phys. Rev. Lett. 119, 131804 (2017).

[56] D. Banerjee et al. (NA64 Collaboration), Phys. Rev. Lett. 118, 011802 (2017).

[57] P. Ade et al. (Simons Observatory Collaboration), J. Cosmol. Astropart. Phys. 02 (2019) 056.

[58] K. N. Abazajian et al. (CMB-S4 Collaboration), arXiv: 1610.02743.

[59] K. Abazajian et al., arXiv:1907.04473.

[60] N. Sabti, J. Alvey, M. Escudero, M. Fairbairn, and D. Blas, J. Cosmol. Astropart. Phys. 01 (2020) 004.

[61] K. K. Boddy and J. Kumar, Phys. Rev. D 92, 023533 (2015).

[62] J. Smirnov and J. F. Beacom, Phys. Rev. Lett. 125, 131301 (2020).

[63] D. Curtin et al., Phys. Rev. D 90, 075004 (2014).

[64] A. Ariga et al. (FASER Collaboration), Phys. Rev. D 99, 095011 (2019).

[65] V. Khachatryan et al. (CMS Collaboration), Phys. Lett. B 752, 146 (2016). 